\title{
First detection and molecular identification of Borrelia garinii isolated from human skin in Taiwan
}

Lyme disease is an emerging tick-borne spirochaetal infection that can cause multisystem human illness. Clinical symptoms vary among infected persons, ranging from a relatively benign skin lesion to severe arthritic, neurological and cardiac manifestations (Steere, 1989). Based on pathogenicity to humans, the causative agent for Lyme disease can be classified into three major genospecies: Borrelia burgdorferi sensu stricto, Borrelia garinii and Borrelia afzelii (group VS461) (Baranton et al., 1992). Although laboratory-diagnosed human cases of Lyme disease have been reported in Taiwan (Shih et al., 1998; Li et al., 2007) and B. burgdorferi spirochaetes also have been isolated from rodents in the Taiwan area (Shih \& Chao, 1998), the causative agent responsible for human Lyme disease has never been isolated or identified from humans in Taiwan to our knowledge.

Genomic analysis of Borrelia isolates by sequence similarity of a specific target gene has proven useful for the species identification of Borrelia spirochaetes. The 5S ( rrf)-23S ( $r r l)$ intergenic spacer amplicon gene is unique and highly conserved in B. burgdorferi sensu lato (Fukunaga \& Sohnaka, 1992), and its diversity is valuable for distinguishing the genetic heterogeneity and clarifying the genetic identity of Borrelia spirochaetes isolated from various biological and geographical sources, including skin specimens from patients (Mathiesen et al., 1997; Rijpkema et al., 1997). In this study, we identified the genospecies of Borrelia spirochaetes isolated from a skin biopsy specimen of an indigenous human case by using a PCR assay targeting the 5S (rrf)$23 \mathrm{~S}(\mathrm{rrl})$ intergenic spacer amplicon gene of B. burgdorferi sensu lato, and clarified the genetic identity by analysing

The GenBank/EMBL/DDBJ accession number for the 5S (rrf)-23S (rrl) intergenic spacer amplicon gene reported in this study is FJ686175. phylogenetic relationships with other Borrelia species.

We conducted an investigation for $B$. burgdorferi infection among patients with clinical manifestations suggestive of Lyme disease. A 26-year-old Taiwanese woman living in the suburban area of Taipei City in northern Taiwan visited the dermal clinic of the National Taiwan University Hospital with the chief complaint of a long-lasting skin lesion observed on the lateral side of her abdomen. Her white blood cell count was $8000 \mathrm{~mm}^{-3}(52 \%$ neutrophils, $21 \%$ lymphocytes, $9 \%$ monocytes), and a normal haemoglobin level $\left(17.9 \mathrm{~g} \mathrm{dl}^{-1}\right)$ and platelet count $\left(212000 \mathrm{~mm}^{-3}\right)$ were observed. The lesion was initially observed for 6 months following a suspected arthropod bite. She had no history of travel to any other countries during the previous 10 years. Based on the clinical features, the skin lesion was diagnosed as morphea (or localized scleroderma) and skin biopsy specimens were collected for cultivation of Borrelia spirochaetes as well as being stored at $-80{ }^{\circ} \mathrm{C}$ for further investigation. Serum specimens from this patient were subsequently collected for serological tests of Lyme disease infection.

For isolation of spirochaetes, skin biopsy specimens from the patient were cleaned with $75 \%$ ethanol solution for 1-2 min and then washed twice in sterile distilled water. Afterwards, an individual skin specimen was dissected into pieces and inoculated into a culture tube containing BSK-H medium (B3528; Sigma) supplemented with $6 \%$ rabbit serum (R7136; Sigma). After incubating at $34{ }^{\circ} \mathrm{C}$ in a humidified incubator with $5 \% \mathrm{CO}_{2}$ (Sanyo Electric Biomedical), skin cultures were examined weekly for evidence of spirochaetes using a dark-field microscope (BX-60; Olympus). For purification of spirochaetes, positive cultures were transferred to new culture tubes by serial dilution and were further filtered with a
$0.45 \mu \mathrm{m}$-pore-size syringe filter (Sartorius), as described previously (Shih \& Chao, 1998).

Serodiagnosis for Lyme disease infection based on a two-step process recommended by the US Centers for Disease Control (CDC, 1997) was performed by an indirect immunofluorescence assay (IFA) followed by Western immunoblot (WB) analysis using paired serum collected from the same patient. Borrelia spirochaetes from cultured isolates were used as antigens for IFA and WB assays, as described previously (Shih \& Chao, 1998). For the IFA test, whole spirochaetes were coated in a 10well glass IFA slide, fixed with acetone, and covered with $20 \mu$ l undiluted patient's serum. This slide was incubated for $1 \mathrm{~h}$ at $37{ }^{\circ} \mathrm{C}$, washed three times with PBS, and allowed to air-dry. Twenty microlitres of FITC-conjugated goat anti-human IgG (catalogue no. F0132; Sigma) and IgM (catalogue no. F5384; Sigma) were used as secondary antibody to determine the seroreactivity against Borrelia spirochaetes, as described previously (Shih \& Chao, 1998). For the WB assay, electrophoresed Borrelia proteins were transferred from the SDS-PAGE gels to nitrocellulose blotting membranes using a semi-dry electroblotter (PhastTransfer electrode cassette; Pharmacia Biotech). The transferred membranes were blocked for $2 \mathrm{~h}$ with $3 \%$ gelatin in Tris-buffered saline (TBS, $\mathrm{pH}$ 7.5) containing $20 \mathrm{mM}$ Tris and $500 \mathrm{mM} \mathrm{NaCl}$, and then incubated for $2 \mathrm{~h}$ at room temperature in a $1: 20$ dilution of the patient's serum. After being washed with buffer solution, the membranes were further immersed for $2 \mathrm{~h}$ in alkaline phosphatase-conjugated goat anti-human IgG (catalogue no. 109-055-008; Jackson ImmunoResearch) and IgM (catalogue no. 109-055-043; Jackson ImmunoResearch) diluted $1: 5000$ with $0.05 \%$ Tween 20 in TBS (T-TBS), as described previously (Shih \& Chao, 1998). The reacted membranes were then washed twice with T-TBS, a substrate solution $(10 \mathrm{ml}$ nitro 
blue tetrazolium; catalogue no. B-5655, Sigma) was added to develop the colour for $1 \mathrm{~min}$, and the reacted membranes were washed with distilled water and airdried for analysis of spirochaetal protein bands.

Total genomic DNA extracted from individual spirochaete-positive cultures by a DNeasy Tissue kit (catalogue no. 69506; Qiagen) was used as template for PCR amplification. A nested PCR assay was performed with primers designed to amplify the variable spacer region between two conserved duplicate structures. A specific primer set corresponding to the $3^{\prime}$ end of the $5 \mathrm{~S}$ rRNA ( $5^{\prime}$-CGACCTTCTTCGCCTTAAAGC- $3^{\prime}$ ) and the $5^{\prime}$ end of the 23S rRNA (5' -TAAGCTGACTAATACTAATTACCC-3') was designed and applied for the primary amplification (Schwartz et al., 1992). In the nested PCR, a primer set of primer 1 (5' -CTGCGAGTTCGCGGGAGA-3') and primer 2 (5'-TCCTAGGCATTCACCATA-3') was used and expected to yield a 226-266 bp fragment depending on the Borrelia strain, as described previously (Postic et al., 1994). PCR amplification included 2 min denaturation at $96{ }^{\circ} \mathrm{C}$, followed by 30 cycles of denaturation at $94{ }^{\circ} \mathrm{C}$ for $30 \mathrm{~s}$, annealing at $55{ }^{\circ} \mathrm{C}$ for $30 \mathrm{~s}$, and extension at $72{ }^{\circ} \mathrm{C}$ for $40 \mathrm{~s}$. Nested PCR was performed under the same conditions except for annealing at $59{ }^{\circ} \mathrm{C}$ for $30 \mathrm{~s}$. After purification (QIAquick PCR Purification kit; catalogue no. 28104), sequencing reactions were performed using the Big Dye Terminator Cycle Sequencing kit (Perkin-Elmer Biosystem) with an ABI Prism 377-96 DNA sequencer (Applied Biosystems). The resulting sequences were aligned with the CLUSTAL $\mathrm{w}$ software and further analysed by comparing with other Borrelia sequences that were available in GenBank. Neighbour-joining and maximumparsimony methods were also used to estimate the phylogeny of the entire alignment using MEGA 4.0 software.

Although in vitro isolation of Borrelia spirochaetes from infected tissues is insensitive, culture of B. burgdorferi sensu lato undoubtedly provides the best confirmation of active infection. Indeed, cultivation of skin biopsy specimens gives the highest success rates for clinical samples (Picken et al., 1998), and successful recovery of $B$. burgdorferi spirochaetes has been reported from patients with late cutaneous manifestations more than 10 years after onset of the skin lesion (Asbrink \& Hovmark, 1985). In the present case, Borrelia spirochaetes were isolated from the skin biopsy specimens more than 6 months after the onset. These observations demonstrate the feasibility of cultivation of Borrelia spirochaetes from skin specimens of a chronic skin lesion presenting as morphea (or localized scleroderma).

Lyme disease infection was also confirmed by the IFA test with a positive reactivity $(>1: 40)$ against Borrelia spirochaetes, and by WB analysis with significant IgG reactivities against the major protein antigens (30, 39, 41, 45, 58, 63 and $66 \mathrm{kDa}$ ) of B. burgdorferi and IgM reactivities against the 39,45 and $66 \mathrm{kDa}$ protein antigens of B. burgdorferi (Fig. 1a). Paired serum from the same patient collected 2 months after antibiotic treatment still revealed IgG banding with the major protein antigens of 45, 63 and $66 \mathrm{kDa}$ of B. burgdorferi, although no IgM reactivity was found (Fig. $1 \mathrm{~b}$ ). The differential WB banding in infected patients is attributed to the variable seroreactivity of the patient's serum against Borrelia protein antigens. Indeed, a previous study on the frequency of WB reactivity to specific Borrelia protein antigens also revealed that seroreactivity of IgG antibody against the higher molecular mass bands ( $41 \mathrm{kDa}$ and above) was most frequently detected in infected patients (Trevejo et al., 2001). Results from the present case also revealed significant seroreactivities of IgG and IgM antibodies (a)

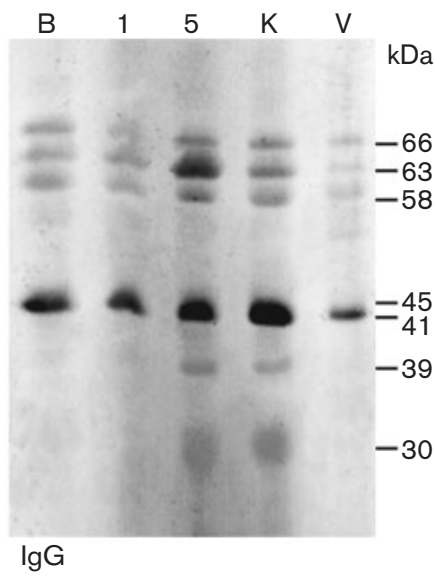

(b)

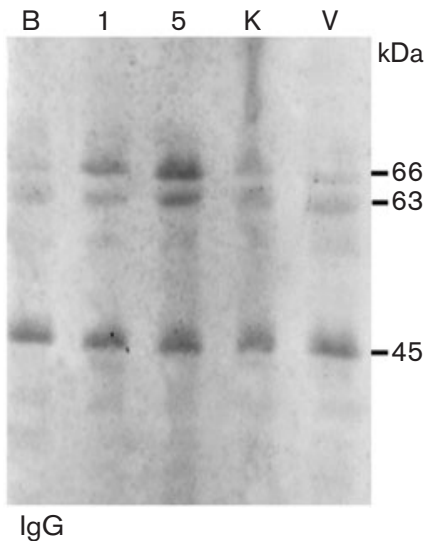

Fig. 1. A comparative Western immunoblot analysis with the patient's serum collected before (a) and 2 months after (b) antibiotic treatment reveals differential seroreactivities against major protein antigens of various strains of $B$. burgdorferi. Lanes: $\mathrm{B}$, American type strain B31 isolate; 1 and 5, Taiwan strain TWKM1 and TWKM5 isolates; K, K48 isolate of $B$. garinii; V, VS461 isolate of $B$. afzelii. 


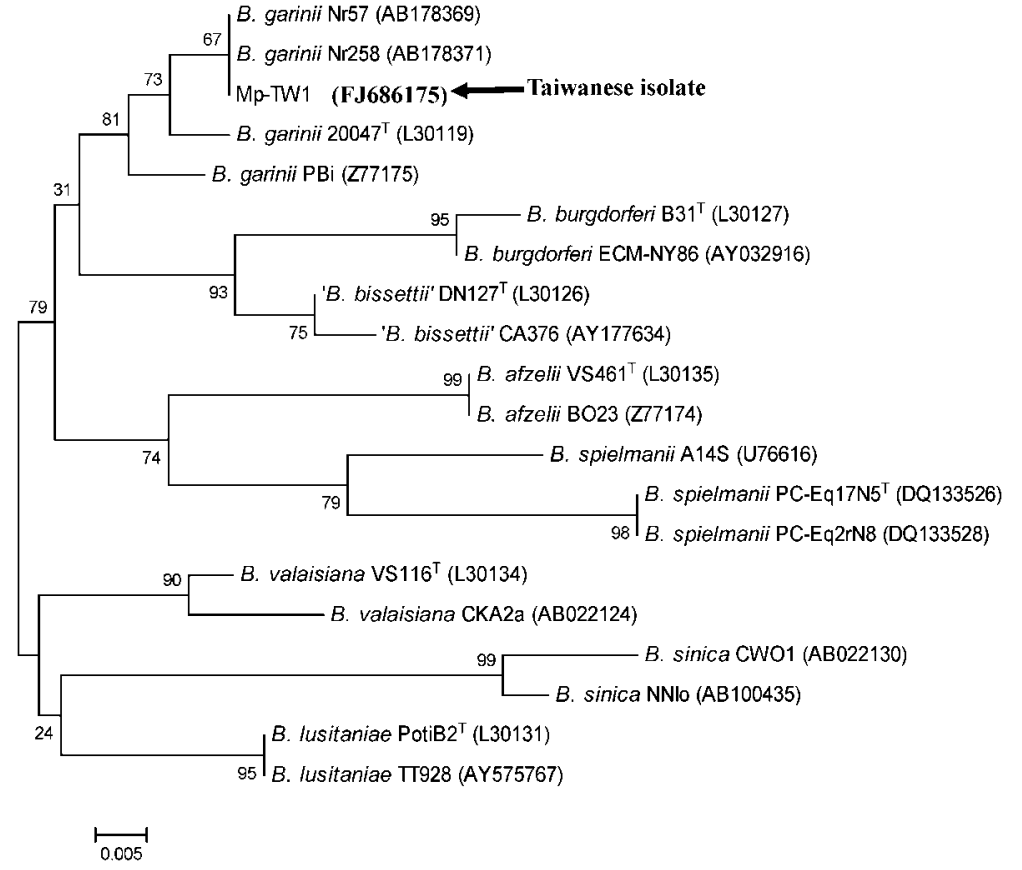

Fig. 2. Phylogenetic relationships based on a comparison of the $5 S(r r f)-23 S(r r)$ rRNA gene sequences between Borrelia strain Mp-TW1 (GenBank accession no. FJ686175) isolated from human skin in Taiwan and 19 other strains of Borrelia spirochaetes. The tree was constructed and analysed by the neighbour-joining method, and was congruent with the maximum-parsimony method (data not shown) using 1000 bootstrap replicates. Numbers at the nodes indicate the percentages of reliability of each branch of the tree. Branch lengths are drawn proportional to the estimated sequence divergence.

against the higher molecular mass bands of Borrelia protein antigens, and the persistence of a positive anti-B. burgdorferi IgG antibody titre after antibiotic therapy with tetracycline $250 \mathrm{mg}$ three times per day for 3 weeks. These observations demonstrate the significance of persistent differential seroreactivity of individual patients against Borrelia protein antigens.

Although PCR amplification of the intergenic spacer region located between the 5S ( rrf) and 23S ( $r r l)$ genes of $B$. burgdorferi sensu lato has been reported to generate a DNA fragment of approximately 226-266 bp (Postic et al., 1994), the size of the nucleotide sequence depends on the strain of Borrelia isolate and may actually represent the genetic distance of phylogenetic divergence between or within the genospecies of Borrelia isolates (Postic et al., 1994; Mathiesen et al., 1997; Rijpkema et al., 1997). In addition to the discovery of human cases of Lyme disease infection in Taiwan, identification of the causative agent responsible for Lyme disease existing in the patient's tissue is required to clarify the transmission cycle for Borrelia spirochaetes in Taiwan. Results from this study indicate that the Borrelia spirochaete isolated from the skin specimen (designated strain Mp-TW1) represents a monophyletic group closely affiliated with the genospecies of B. garinii, and all the $B$. garinii spirochaetes constitute a separate clade distinguished clearly from other Borrelia genospecies by both neighbourjoining (Fig. 2) and maximum-parsimony (data not shown) methods. Further investigations focused on the detection of Borrelia spirochaetes among patients characterized with various clinical manifestations would help to elucidate the genetic diversity of the genospecies of Borrelia spirochaetes in the Taiwan area.

In conclusion, our finding provides the first convincing evidence regarding the identification of B. garinii isolated from human skin in Taiwan. The genetic identity of this spirochaete was confirmed by analysing sequence homology and phylogenetic analysis of the 5S (rrf)-23S $(r r l)$ intergenic spacer amplicon gene. Further investigations focused on the genetic variability of $5 \mathrm{~S}(\mathrm{rrf})-23 \mathrm{~S}(\mathrm{rrl})$ intergenic spacer amplicon genes among Borrelia spirochaetes isolated from patients, vector ticks and reservoir hosts may provide a better understanding of the significance of the genetic diversity of Borrelia spirochaetes in relation to their pathogenicity for Lyme disease infection in Taiwan.

\section{Acknowledgements}

This work was supported in part by grants from the National Science Council (NSC962314-B-016-025-MY3) and Department of Health (DOH91-DC-1066), The Executive Yuan, Taipei, Taiwan, Republic of China.

\section{Li-Lian Chao, ${ }^{1}$ Yi-Ju Chen ${ }^{2}$ and Chien-Ming Shih ${ }^{1}$}

${ }^{1}$ Department of Parasitology and Tropical Medicine, National Defense Medical

Center, Taipei, Taiwan, Republic of China

${ }^{2}$ Department of Dermatology, National Taiwan University Hospital, Taipei, Taiwan, Republic of China

Correspondence: Chien-Ming Shih (cmshih@ndmctsgh.edu.tw)

Asbrink, E. \& Hovmark, A. (1985). Successful cultivation of spirochetes from skin lesions of patients with erythema chronicum migrans Afzelius and acrodermatitis chronica atrophicans. Acta Pathol Microbiol Immunol Scand [B] 93, 161-163.

Baranton, G., Postic, D., Saint Girons, I., Boerlin, P., Piffarretti, J. C., Assous, M. \& Grimont, P. A. D. (1992). Delineation of Borrelia burgdorferi sensu stricto, Borrelia garinii sp. nov., and group VS461 associated with Lyme borreliosis. Int J Syst Bacteriol 42, 378-383.

CDC (1997). Case definitions for infectious conditions under public health surveillance. MMWR Morb Mortal Wkly Rep 46, 20-21.

Fukunaga, M. \& Sohnaka, M. (1992). Tandem repeat of the $23 \mathrm{~S}$ and $5 \mathrm{~S}$ ribosomal RNA genes in Borrelia burgdorferi, the etiological agent of Lyme disease. Biochem Biophys Res Commun 183, 952-957.

Li, T. H., Shih, C. M., Lin, W. J., Lu, C. W., Chao, L. L. \& Wang, C. C. (2007). Erythema migrans mimicking cervical cellulitis with deep neck infection in a child with Lyme disease. J Formos Med Assoc 106, 577-581. 
Mathiesen, D. A., Oliver, J. H., Kolbert, C. P., Tullson, E. D., Johnson, B. J. B., Campbell, G. L., Mitchell, P. D., Reed, K. D., Telford, S. R., III \& other authors (1997). Genetic heterogeneity of Borrelia burgdorferi in the United States. J Infect Dis 175, 98-107.

Picken, R. N., Strle, F., Picken, M. M., RuzicSabljic, E., Maraspin, V., Lotric-Furlan, S. \& Cimperman, J. (1998). Identification of three species of Borrelia burgdorferi sensu lato (Borrelia burgdorferi sensu stricto, B. garinii, and $B$. afzelii) among isolates from acrodermatitis chronica atrophicans lesions. J Invest Dermatol 110, 211-214.

Postic, D., Assous, M. V., Grimont, P. A. \& Baranton, G. (1994). Diversity of Borrelia burgdorferi sensu lato evidenced by restriction fragment length polymorphism of $r r f(5 S)$ $\operatorname{rrl}(23 \mathrm{~S})$ intergenic spacer amplicons. Int J Syst Bacteriol 44, 743-752.

Rijpkema, S. G., Tazelaar, D. J., Molkenboer, M., Noordhoek, G. T., Plantinga, G., Schouls, L. M. \& Schellekens, J. F. (1997). Detection of Borrelia afzelii, Borrelia burgdorferi sensu stricto, Borrelia garinii and group VS116 by PCR in skin biopsies of patients with erythema migrans and acrodermatitis chronica atrophicans. Clin Microbiol Infect 3, 109-116.

Schwartz, J. J., Gazumyan, A. \& Schwartz, I. (1992). rRNA gene organization in the Lyme disease spirochete, Borrelia burgdorferi. J Bacteriol 174, 3757-3765.
Shih, C. M. \& Chao, L. L. (1998). Lyme disease in Taiwan: primary isolation of Borrelia burgdorferi-like spirochetes from rodents in the Taiwan area. Am J Trop Med Hyg 59, 687-692.

Shih, C. M., Wang, J. C., Chao, L. L. \& Wu, T. N. (1998). Lyme disease in Taiwan: first human patient with characteristic erythema chronicum migrans skin lesion. J Clin Microbiol 36, 807-808.

Steere, A. C. (1989). Lyme disease. $N$ Engl J Med 321, 586-596.

Trevejo, R. T., Krause, P. J., Schriefer, M. E. \& Dennis, D. T. (2001). Evaluation of a two-test serodiagnostic method for community assessment of Lyme disease in an endemic area. Am J Trop Med Hyg 65, 563-566. 